\title{
Using Electrical Resistance Change to Monitor the Damage in a Thick CFRP Plate Caused by a Dent*
}

\author{
Akira TODOROKI**, Yasuhiro SHIMAZU***, Yosihiro MIZUTANI**** \\ and Ryosuke MATSUZAKI**** \\ ** Department of Mechanical Sciences and Engineering \\ Tokyo Institute of Technology \\ 2-12-1, O-okayama, Meguro, Tokyo 152-8552, Japan, \\ E-mail:atodorok@ginza.mes.titech.ac.jp \\ *** Tokyo Institute of Technology \\ **** Department of Mechanical Sciences and Engineering \\ Tokyo Institute of Technology
}

\begin{abstract}
An impact load on a thick carbon fiber reinforced plastic (CFRP) plate usually causes a dent. The dent results in a decrease of electrical resistance. Our previous paper demonstrated the effect of the dent in the measurement of electrical resistance change of a thick CFRP plate subjected to impact loading. The present paper focuses on the electrical resistance decrease caused by the dent. Numerous FEM analyses were performed, and the relationship between electrical resistance changes and delamination location was constructed, by measuring the response surfaces. Several experiments were conducted and the measured electrical resistance changes were used to predict damage location from the response surfaces. The present study shows that the dent decreases the electrical resistance change ratio even in the adjacent segments, and the electrical resistance changes caused by the dent enable us to monitor the damage location. FEM analyses for a projected area model are sufficient to construct the relationships without resorting to many experiments.
\end{abstract}

Key words: Composites, CFRP, Damage, Delamination, Electrical Resistance, Dent, FEM

\section{Introduction}

The electrical resistance change method is one of the health monitoring methods for carbon fiber reinforced plastics (CFRP) laminated composites. Many researchers have published applications of the electrical resistance change method for damage detection of these composites ${ }^{(1)-(8)}$. We have been doing continuous research on the electrical resistance change method for damage monitoring ${ }^{(9)-(17)}$.

For thin CFRP laminates ${ }^{(16)}$, delamination cracking can occur for lower indentation loads. The delamination cracks cause an electrical resistance increase because a delamination crack impedes the electric current path. For thick CFRP laminates (17), however, damage occurs at higher indentation loads. When the indentation load is high, a dent is made with delamination cracking. The dent causes a decrease in electrical resistance in the thickness direction.

The present study focuses on thick CFRP laminated composites because they are usually adopted for aerospace components. For thick CFRP laminated composites, a dent

*Received 14 Sep., 2010 (№. 10-0418) [DOI: 10.1299/jmmp.5.44]

Copyright $@ 2011$ by JSME 
made by an impact, or an indentation load with matrix cracking and multiple delamination cracks, causes the electrical resistance to decrease, as shown in the previous study ${ }^{(17)}$. However when measuring the electrical resistance changes of multiple segments in a thick CFRP laminated composite plate with multiple electrodes, the effect on the other segments of a dent in a particular segment is not known. In the present study, experiments are performed to examine this effect using thick CFRP plates with multiple electrodes.

To monitor the location of the damage in the laminated CFRP plate, the relationship between the measured electrical resistance changes and the delamination location must be obtained. In our previous studies ${ }^{(10)-(14)}$, many experiments were conducted and the least-squares-error method was adopted to obtain the response surfaces: the response is the delamination location and the variables are the measured electrical resistance changes. In reality, using laminated CFRP, the experimental cost is quite high.

To reduce these experimental costs, FEM analyses were adopted to obtain the relationship. In reality the actual damage to the laminated CFRP has complicated multiple delamination cracks ${ }^{(18)}$. It is impossible to analyze the multiple delamination cracks in detail using 3-D FEM analyses. In the present study, therefore, two types of simplified models are proposed and compared with the experimental results. The response surfaces were determined and damage monitoring was performed for the experimental results using the FEM analyses.

\section{Specimens and experiments}

The material used in the present study was unidirectional carbon/epoxy prepreg produced by Mitsubishi Rayon Co. Ltd. The prepreg production number was PYROFIL \#380. As a thick CFRP laminate test piece, a simple sixteen-ply quasi-isotropic laminate with stacking sequence $\left[(0 / 90 / 45 /-45)_{2}\right] \mathrm{s}$ was used. The CFRP laminates were cured at $130^{\circ} \mathrm{C}$ and $0.6 \mathrm{MPa}$ for $90 \mathrm{~min}$. using an autoclave. Plate type specimens $170 \mathrm{~mm}$ long and $84 \mathrm{~mm}$ wide were made from the CFRP plate as shown in Fig.1. In Fig.1, (a) and (b) are the indentation points describer later. The thickness of the specimens was approximately $4 \mathrm{~mm}$. and the fiber volume fraction, $V_{\mathrm{f}}$, was 0.53 . In total, 12 electrodes were mounted on the dual surface of the CFRP beam to obtain an oblique path of electric current flow. The process used to make the electrodes was shown in a previous study ${ }^{(19)}$.

To make a delamination crack in the CFRP plate, an indentation test was used. The test jig is illustrated in Fig.2; the diameter of the $60 \mathrm{~mm}$ metal cylinder was used as a support. The multiple delamination cracks were observed using an ultrasonic C-scan SDS-5400R made by Krautkramer Japan Co. Ltd. At the indentation point, a piece of tire rubber (thickness $=1 \mathrm{~mm}$ ) was used to prevent fiber breakages in the CFRP laminated plate and to retain the electric insulation. At the cylinder support, a one millimeter thick Teflon film was inserted to prevent electrical contact between the specimen and the metallic jig. The loading speed of the indentation test was $0.5 \mathrm{~mm} / \mathrm{min}$. Coordinates $x-y-z$ were defined as shown in Fig.1.

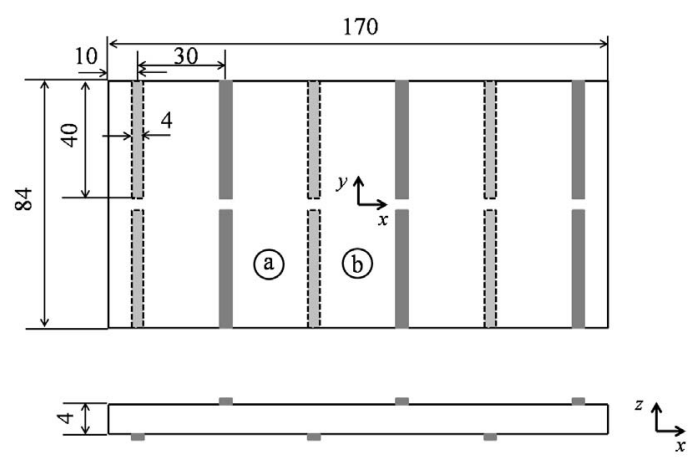

Fig.1 Specimen configuration 
The electrical resistance change was measured using a static strain data logger (Kyowa Electronic Instruments Co. LTD.: UCAM-10A). A two-probe method was used to measure the electric resistance change with a bridge circuit shown in Fig. 3.

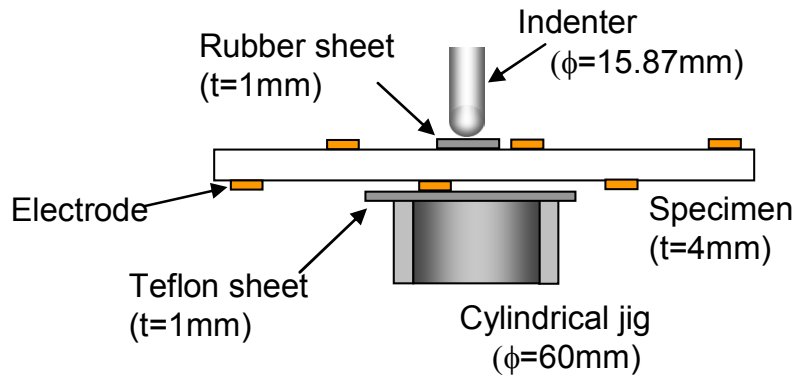

Fig.2 Indentation test method to make delamination cracks

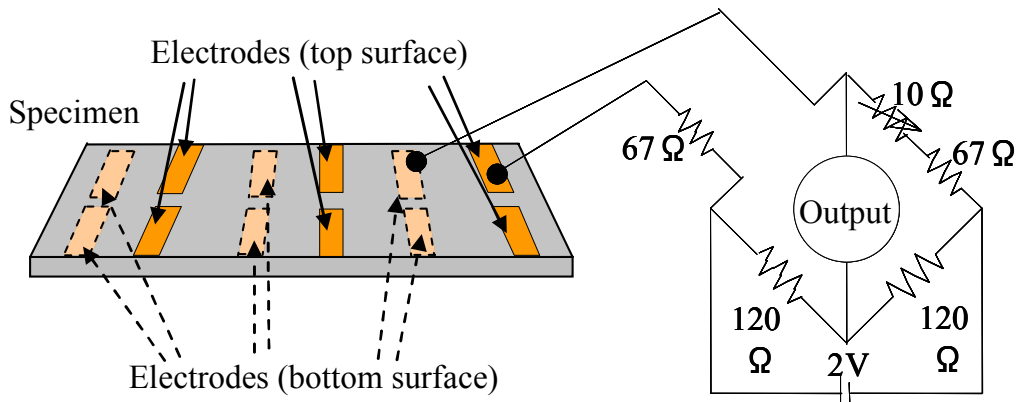

Fig. 3 Bridge circuit used to measure electric resistance change

The indentation method shown in Fig.2 generates multiple delamination cracks with a single dent impact. Typical dent configurations have already been shown in a previous paper ${ }^{(17)}$. Figure 4 shows a typical dent made by a tool drop. The dent was observed using a three-dimensional LASER profilometer (Keyence, Type KS-1100) ${ }^{(17)}$. Figure 5 shows the ultrasonic C-scan image of the multiple delamination cracks.

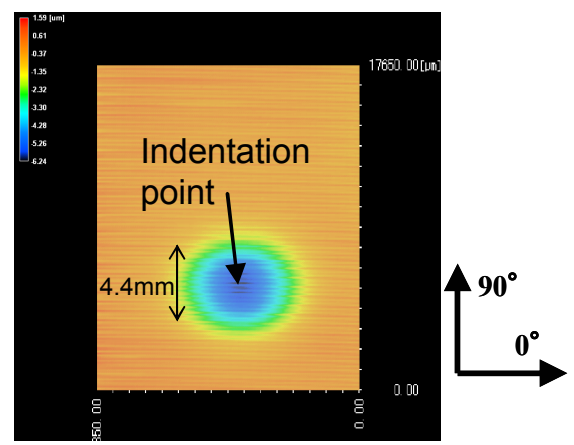

Fig. 4 Typical dent configuration measured by using the LASER profilometer ${ }^{(17)}$ (Depth approximately $5 \mu \mathrm{m}$ )


Fig.5 Typical delamination C-scan image 


\section{Monitoring method}

To monitor damage location, the electrical resistance change ratios of multiple segments must be measured. In the present study, electrodes were mounted on the top and bottom surfaces of the thick CFRP plate, and the electric current flowed in an oblique path through the thickness of the plate. Since 12 electrodes were mounted in two lines (six electrodes per line), there were 10 segments. We defined the normalized electric resistance change ratio $\left(r_{\mathrm{i}}\right)$ as $r_{\mathrm{i}}=\left(\Delta R_{\mathrm{i}} / R_{0 \mathrm{i}}\right) / L$ for each segment $\left(\Delta R_{\mathrm{i}}\right.$ is the electrical resistance change of $i$ th segment; $R_{0 \mathrm{i}}$ is the initial electrical resistance of the $i$ th segment; the norm $L$ is $\left.L=\sqrt{\left(\Delta R_{1} / R_{01}\right)^{2}+\cdots+\left(\Delta R_{10} / R_{010}\right)^{2}} ; i=1, \ldots, 10\right)$. As in previous papers ${ }^{(10)-(15)}$, the response surfaces of quadratic polynomials are adopted to obtain the relationships between the electrical resistance changes and the delamination locations.

Let us consider the $x$ and $y$ coordinates as shown in Fig.1. The delamination location $(x, y)$ can be obtained using the two response surfaces as follows:

$$
\begin{aligned}
& x=\beta_{x 0}+\beta_{x x} r_{1}+\cdots \beta_{x 10} r_{01}+\beta_{x 11} r_{1}^{2}+\cdots \beta_{x 20} r_{10}^{2}+\beta_{x 21} r_{1} r_{2} \cdots \beta_{x 65} r_{9} r_{10} \\
& y=\beta_{y 0}+\beta_{y 1} r_{1}+\cdots \beta_{y 10} r_{01}+\beta_{y 11} r_{1}^{2}+\cdots \beta_{y 20} r_{10}^{2}+\beta_{y 21} r_{1} r_{2} \cdots \beta_{y 65} r_{9} r_{10}
\end{aligned}
$$

The 66 unknown coefficients $\beta_{i j}(\mathrm{i}=x$ or $y, \mathrm{j}=0 \ldots 65$ ) were determined using the least-squares-error method. To obtain better estimates using the least-squares-error method, we needed approximately twice the number of data sets compared with the number of unknown coefficients. In the present study, we needed approximately 130 data sets. To reduce the number of experiments, FEM analyses were adopted. First, the electrical conductance of the CFRP was measured. Since the method uses the normalized electrical resistance change ratio, the absolute values are not important but the relative values are significant for the FEM analyses. The quadratic polynomials are selected here because the quadratic polynomial was sufficient for the delamination monitoring in the previous studies ${ }^{(10)-(15)}$.

In the present study, 55 FEM analyses for various locations were performed. In addition to the FEM analyses, 110 new sets of data with noise were made by adding $5 \%$ noise to the FEM analyses. The total of 165 data sets was used to obtain the relationships.

The measured experimental results were inserted into Eqs. (1) and (2). From this, the damage location $(x, y)$ was obtained.

In the present study, the dimensions were almost the same for all damage because the indentation loads were almost the same. This means the smallest delamination cracks were used for monitoring to avoid fiber breakages, and therefore, the damage dimensions were not dealt with.

\section{FEM analyses}

It is impossible to analyze the actual complicated delamination cracks from a dent using FEM. Therefore, two models are proposed: the projected model and the multi-crack model. The two models include a high-conductive square pole which is a simplified dent effect: the pole has higher conductivity in the thickness direction compared with the normal CFRP. The two models are shown in Figs.6 (a) and (b). Each model has a highly conductive square pole as shown in Fig.6. From observations, the dimensions of the dent were decided at $4 \mathrm{~mm}$ square, and the dent was located in the middle of the delamination crack, shown as the bright orange area of the C-scan image in Fig.5.

As mentioned in the reference ${ }^{(20)}$, the delamination cracks usually locate in a helical pattern. In the present study, to simplify the model of the multi-crack effect, the helical pattern is not adopted here. The helical pattern may improve the accuracy of the FEM analysis after finding the exact effect of the dent. This is our future work. 


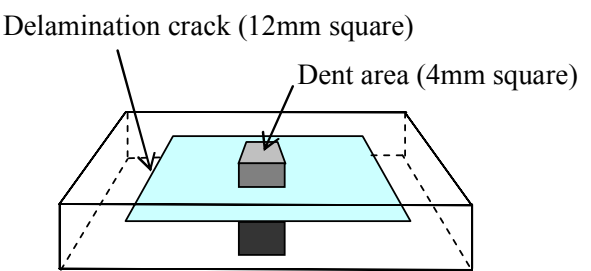

(a) Projected model

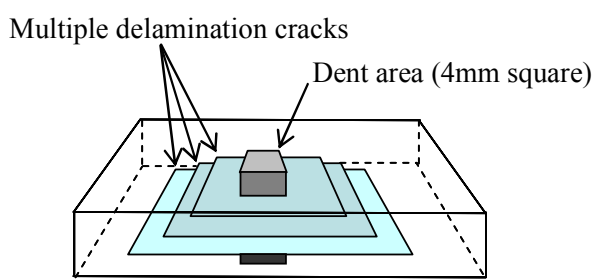

(b) Multi-crack model

Fig. 6 Two models adopted in the present study

The projected model has a large single delamination crack in the projected area as shown in Fig.6(a). The delamination crack area has doubly defined FEM nodes. The release of the two connected nodes enables us to simulate the electric current insulation. In the dent area, there is no delamination crack similar to the observed delamination cracks in Fig.6(b). Since the square pole has high electric conductivity in the thickness direction, the dent causes the electrical resistance to decrease. As we did not know the exact effect of the dent in the conductivity, we used a fitting method here. The delamination crack causes electrical resistance to increase, and the dent causes a decrease. By changing the conductivity at the dent area, we can find the approximate estimated value of the conductivity compared with the experimental results. In the present study, one experimental result was used to find the approximate conductivity in the thickness direction in the dent area. In the other areas, the measured electrical conductivities were used. These values are shown in Table 1.

Table 1 Electric conductivity of CFRP used in FEM analyses

\begin{tabular}{cccc}
\hline$\sigma_{0}(S / m)$ & $\sigma_{90} / \sigma_{0}$ & $\sigma_{t} / \sigma_{0}$ & $\sigma_{t, \text { Dent }} / \sigma_{0}$ \\
\hline 4100 & $8.1 \times 10^{-4}$ & $8.1 \times 10^{-4}$ & $1.3 \times 10^{-3}$ \\
\hline
\end{tabular}

$\sigma_{0}$ is the conductance of fiber direction; $\sigma_{90}$ is the conductance of the transverse direction; $\sigma_{\mathrm{t}}$ is the conductance of the thickness direction and the $\sigma_{\mathrm{t} \text {,Dent }}$ is the conductance in the thickness direction at the dent area.

In a previous paper ${ }^{(17)}$, we found that the actual delamination crack dimensions increase with the increase of depth from the indentation surface. In the present study, multiple delamination cracks are modeled in the multi-crack model as shown in Fig.6 (b). Each interlaminate has a delamination crack. The bottom delamination crack dimension is the same as for the projected model (12 mm square). The first delamination crack dimension was 0 . The dimensions of the other delamination crack increased almost linearly with the depth.

The mesh size of the 3 -D FEM was $2 \times 2 \times 0.25(\mathrm{~mm})$. The total number of nodes was 416,672 , and the total number of elements was 57,120. ANSYS ver.11 was used for the analyses. To obtain the electrical resistance change ratio between adjacent oblique electrodes, a constant electric current of $1 \mathrm{~A}$ was applied to the electrode and voltage was set to 0 at the other electrode.

After each experiment, we obtained the electrical resistance change for every segment. Since the dent area was obtained experimentally, we could calculate the dent effect by changing the conductivity in the thickness direction at the dent square pole using FEM analysis. Using the trial and error method, the conductivity in the thickness direction in the dent square pole was determined. The determined value is shown in Table 1. 


\section{Results and discussion}

\subsection{Electrical resistance change caused by a dent}

Measured normalized electrical resistance change ratio is shown in Fig.7 in which the abscissa is the segment, and the ordinate is the normalized electrical resistance change ratio. The 1-2 means the electrical resistance change ratio measured between electrodes 1 and 2 . In this test, an indentation test was performed at $x=-30 \mathrm{~mm}$, and $y=-22 \mathrm{~mm}$ (the segment " $2-3$ "). The measured results show that the electrical resistance change ratio decreased after the indentation test, as in a previous paper ${ }^{(17)}$. The decrease of the electrical resistance change ratio was observed for every segment. The decrease was the most significant for the segment where the indentation test was performed. Even in the other segment lines such as $7-8,8-9,9-10,10-11$ and 11-12, a small decrease was observed. This means the dent effect was quite significant even if the dent was very small compared with the area of the wide CFRP plate.

To obtain the approximate value of the increased electric conductivity in the thickness direction at the dent square pole, trial and error searches were done using the change of the conductivity. As a result, the conductivity shown in Table 1 was obtained.

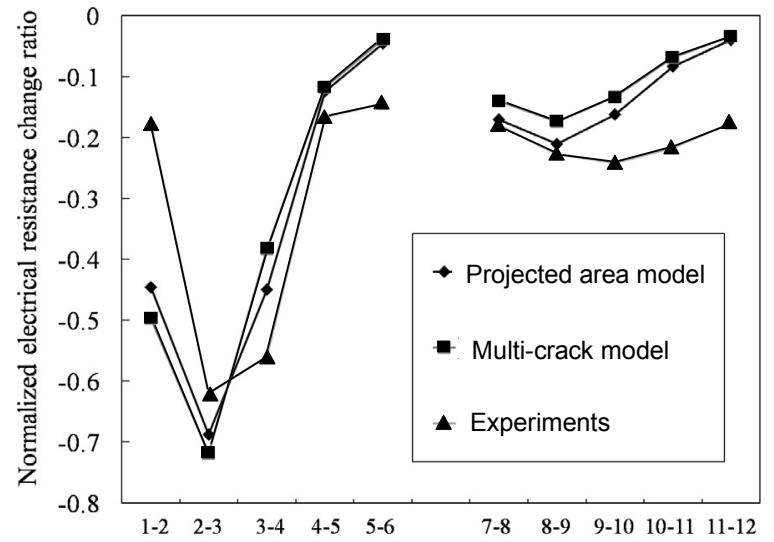

Fig.7 Normalized electrical resistance change ratio (the dent locates in the point (a)).

Since the projected maximum delamination dimension is approximately $12-15 \mathrm{~mm}$, a $12 \mathrm{~mm}$ delamination crack was used for the FEM calculations. Diamond symbols show the results of the electrical resistance change calculated using the FEM with a delamination crack of the projected area model (12 $\mathrm{mm}$ square crack). Square symbols show the results of the electrical resistance change ratio calculated using the FEM with the multiple delamination crack model (maximum size is $12 \mathrm{~mm}$ ). These two models show similar electrical resistance change ratios compared with the experimental results.

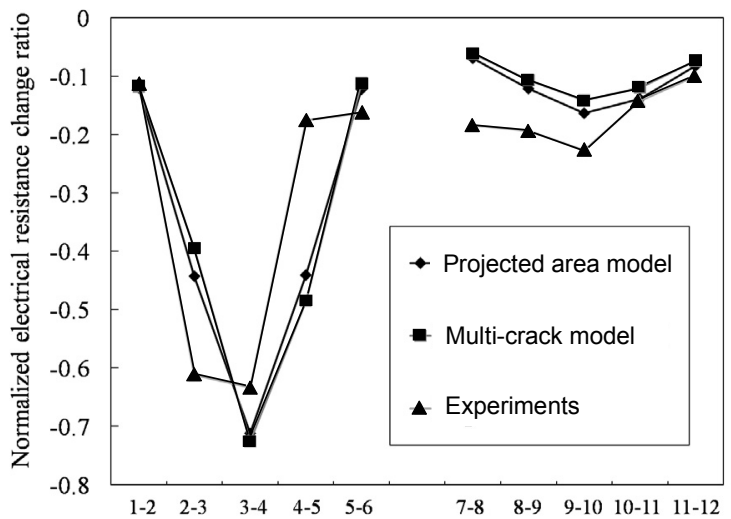

Fig. 8 Normalized electrical resistance change ratio (the dent locates in the point (b)) 
Figure 8 shows the other results of the electrical resistance change ratio: the indentation test was performed at $x=0 \mathrm{~mm}$ and $y=-22 \mathrm{~mm}$ (segment 3-4). The experimental results are shown as triangular symbols. As in the previous results, the electrical resistance change ratio decreases for all segments. From these results, even though the diameter of the dent is small, the electrical effect in the thickness direction is significantly large for all segments, especially adjacent segments. We cannot ignore the effect of the dent for the electrical resistance change method of the CFRP plate.

The FEM results of the projected area model are shown as diamond symbols and the square symbols show the results for the multi-crack model. These results show that the difference between them is quite small.

\subsection{Damage monitoring using electrical resistance change caused by a dent}

Using the measured electric conductivities shown in Table 1, we can calculate the electrical resistance change ratio for many cases. Since the smallest delamination dimension obtained in the indentation experiments was approximately $12 \mathrm{~mm}$, the delamination dimension was fixed to $12 \mathrm{~mm}$. The 55 cases for the different locations were analyzed using the two models: the projected area model and the multi-crack model. Since the FEM analysis was free from experimental noise, random noise was added to the FEM results and 110 sets of artificial experimental data with noise of $5 \%$ of the maximum signal, were made. Using the total 165 data sets, the response surfaces to predict the damage location of the $x$ - and $y$-coordinates were constructed.

As shown in a previous paper ${ }^{(14)}$, when we use the normalized electrical resistance change ratio shown in Eqs. (1) and (2), the response surfaces achieve a very high performance. Eight experimental results were inserted into Eqs. (1) and (2) to obtain the estimated results of the damage locations. Note that every experimental data set was new for the response surfaces.

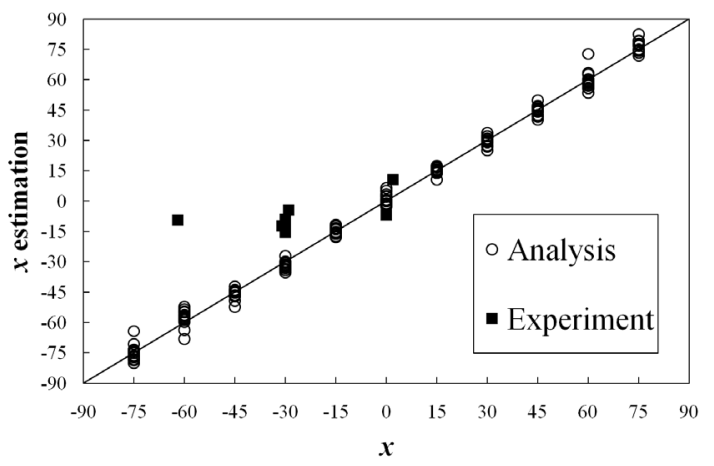

(a) $x$-coordinate estimations

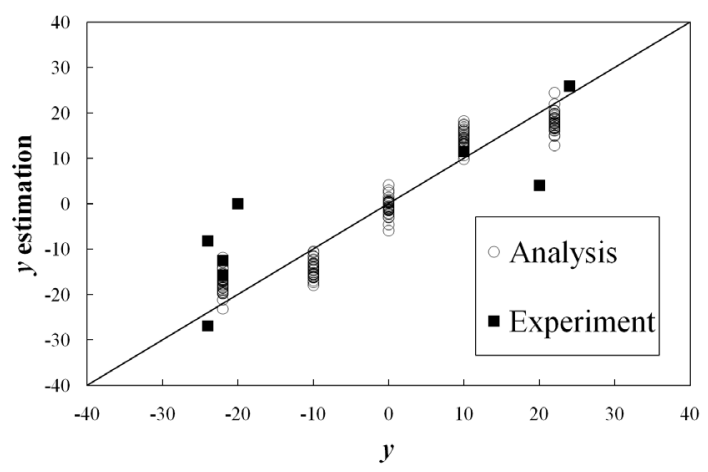

(b) $y$-coordinate estimations

Fig.9 Damage estimation results using electrical resistance change ratios with dent

(Projected area model) 
Figures 9 (a) and (b) show the results of the estimates based on the FEM analyses with the projected area model. The abscissa is the measured indenter location and the ordinate is the estimated indenter location. Open circle symbols represent the estimated results of the FEM analyses used to make the response surfaces. Solid square symbols represent the estimated results for the experimental data. Estimates of the experimental data for the $x$-direction (see Fig.9 (a)) show good results while the estimates for the $y$-results are not as good.

Since there are only two lines of electrodes in the $y$-direction, the estimated results for the experimental data are not as good compared with the estimated results for the $x$-direction. These estimated performances are similar to the results of previous research on the thin CFRP plate ${ }^{(12)}$.

The estimates in the x-direction provide high performance as shown in Fig.9 (a), and this means that we can monitor the damage location from the electrical resistance caused by a dent in a thick CFRP plate.

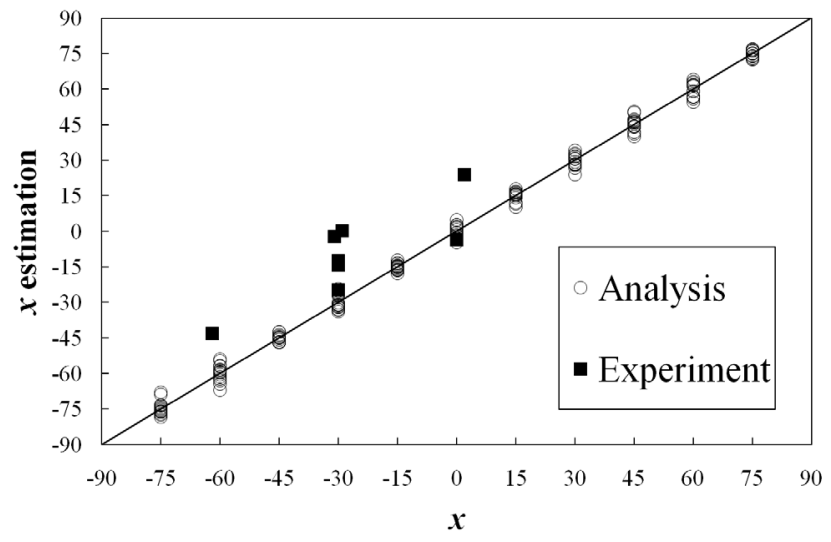

(a) $x$-coordinate estimations

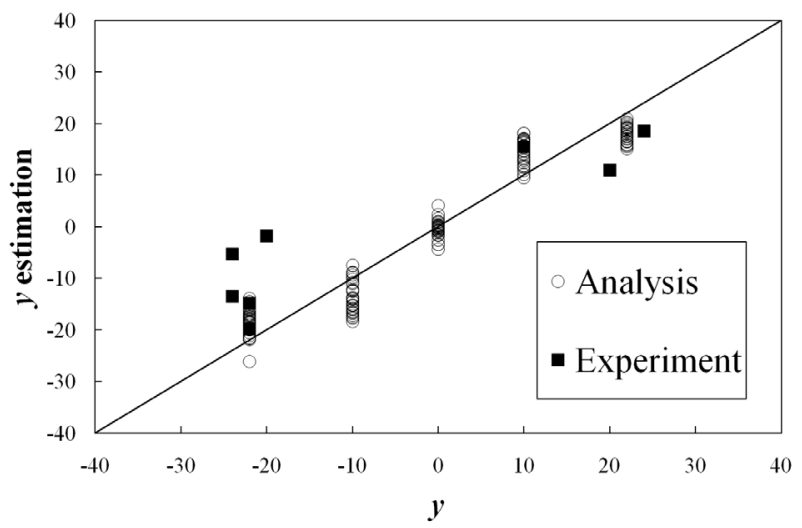

(b) $y$-coordinate estimations

Fig.10 Damage estimation results using electrical resistance change ratios with dent (Multi-crack model)

Figures 10 (a) and (b) show the estimated results of damage locations using FEM analyses with the multi-crack model. As previously mentioned, there is only small difference between the electrical resistance change ratios for the two models. However, the estimated results of the $x$-coordinate of the multi-crack model are better. To compare exactly, the square sum of the errors for both cases are calculated for the eight experimental results. 
Table 2 Square sum of the errors for the estimates for both models

\begin{tabular}{lcc}
\hline & $x$-coordinate & $y$-coordinate \\
\hline Projected area model & 4806 & 1048 \\
\hline Multi-crack model & 3101 & 987 \\
\hline
\end{tabular}

Table 2 shows the results of the square sum of errors for both models. The results of the square sum of errors show that the estimates using the multi-crack model give better performance (small errors) compared with the estimates using the projected area model. The difference is, however, not large. Since the FEM modeling of the multi-crack model is very time consuming and the improvement of the estimation performance is not as high, we can conclude the projected area model is adequate for practical damage monitoring.

\section{Conclusions}

In the present study, the electrical resistance changes of thick CFRP plates with multiple electrodes were measured experimentally, and the effect of a dent on adjacent segments was experimentally investigated. Two models of the complicated delamination crack were used to estimate the electrical resistance changes in FEM analyses: a projected area model and a multi-crack model. The estimates of the decrease of conductivity in the dent area were evaluated using a trial and error search of the experimental results. Fifty five FEM analyses were conducted, and response surfaces were constructed to estimate the damage locations. The results obtained were as follows.

(1) A dent caused by the indentation load causes significant increase in electric conductivity increase in the thickness direction. This dent results in a decrease in electrical resistance even in the adjacent segments in a large plate.

(2) Using the decrease in electrical resistance caused by the dent, it is possible to monitor damage location.

(3) For damage monitoring, a projected area model is simple, and gives affordable performance for monitoring the location of the damage.

\section{References}

(1) K. Schulte, and Ch. Baron, Load and failure analyses of CFRP laminates by means of electrical resistivity measurement, Composites Science and Technology, Vol.36, No.1, (1989) pp.63-76.

(2) N. Muto, H. Yanagida, T. Nakatsuji, M. Sugita, and Y. Ohtsuka, Preventing fatal fractures in carbon-fiber glass-fiber-reinforced plastic composites by monitoring change in electrical resistance, J. Am. Ceram. Soc., Vol.76, No.4, (1993), pp.875-879.

(3) X. Wang and D.D.L. Chung, Sensing delamination in a carbon fiber polymer-matrix composite during fatigue by electrical resistance measurement, Polymer Composites, Vol.18, No.6,(1997), pp.692-700.

(4) P.E. Irving and C.Thiagarajan, Fatigue damage characterization in carbon fibre composite materials using an electrical potential technique, Smart materials and structures, Vol.7, No.4, (1998), pp.456-466.

(5) J.C. Abry, S.Bochard, A. Chateauminois, M. Salvia and G. Giraud, In situ detection of damage in CFRP laminates by electric resistance measurements, Composites Science and Technology, Vol.59, No.6, (1999), pp.929-935.

(6) D.C. Seo and J.J. Lee, Damage detection of CFRP laminates using electrical resistance measurement and neural network, Composite structures, Vol.47, No.1-4, (1999), pp.525-530.

(7) J. B. Park, T. Okabe, N. Takeda and W. A. Curtin, Electromechanical modeling of 
unidirectional CFRP composites under tensile loading condition, Composites Part A, Vol.33, No.2, (2002), pp.267-275.

(8) K. Ogi and Y. Takao, Characterization of piezoresistance behavior in a CFRP unidirectional laminate, Composites Science and Technology, Vol.65, No.2, (2005), pp.231-239.

(9) A. Todoroki, K. Matsuura, and H. Kobayashi, Application of Electric Potential Method to Smart Composite Structures for Detecting Delamination, JSME International J., Series A, Vol.38, No.4, (1995),pp.524-530.

(10) A. Todoroki, Effect of number of electrodes and diagnostic tool for delamination monitoring of graphite/epoxy laminates using electric resistance change, Composites Science and Technology, Vol.61, No.13, (2001),pp.1871-1880.

(11) A. Todoroki, and Y. Tanaka, Delamination identification of cross-ply graphite/epoxy composite beams using electric resistance change method, Composites Science and Technology, Vol.62, No.5, (2002), pp.629-639.

(12) A. Todoroki, Y. Tanaka, and Y. Shimamura, Delamination monitoring of graphite/epoxy laminated composite plate of electric resistance change method, Composites Science and Technology, Vol.62, No.9, (2002), pp.1151-1160.

(13) A. Todoroki, M. Tanaka, and Y. Shimamura, Measurement of orthotropic electric conductance of CFRP laminates and analysis of the effect on delamination monitoring with electric resistance change method, Composites Science and Technology, Vol.62, No.5, (2002), pp.619-628.

(14) A. Todoroki, M. Tanaka and Y. Shimamura, High performance estimations of delamination of graphite/epoxy laminates with electric resistance change method, Composites Science and Technology, Vol.63, No.13, (2003), pp.1911-1920.

(15) A. Todoroki and M. Ueda, Low Cost Delamination Monitoring of CFRP Beams Using Electrical Resistance Changes With Neural Networks, Smart Materials and Structures, Vol.15, No.4, (2006), N75-N85.

(16) A. Todoroki, Y. Samejima, Y. Hirano, R. Matsuzaki and Y. Mizutani, Mechanism of electrical resistance change of a thin CFRP beam after delamination cracking, J. of Solid Mechanics and Materials Engineering, JSME, Vol.4, No.1, (2010), pp.1-11.

(17) A. Todoroki, Y. Samejima, Y. Hirano, R. Matsuzaki and Y. Mizutani, Electrical Resistance Change of Thick CFRP Laminate for Self-Sensing, Journal of Solid Mechanics and Materials Engineering, JSME, ,Vol.4, No.6, (2010) pp.658-668.

(18) H. Suemasu, Effects of multiple delaminations on compressive buckling behaviors of composite panels, J. Composite Materials, Vol.27, No.12, (1993), pp1172-1192.

(19) A. Todoroki, K. Suzuki, Y. Mizutani and R. Matsuzaki, Durability Estimates of Copper Plated Electrodes for Self-sensing CFRP Composites, Journal of Solid Mechanics and Materials Engineering, JSME, Vol.4, No.6, (2010).pp.610-620.

(20) H. Suemasu, Effects of multiple delaminations on compressive buckling behaviors of composite panels, J. Composite Materials, Vol.27, No.12, (1993) pp1172-1192. 